\title{
A psicologia e a educação ambiental ${ }^{*}$
}

\author{
Luana dos Santos Raymundo ${ }^{l}$ \\ Ariane Kuhnen ${ }^{2}$ \\ Universidade Federal de Santa Catarina
}

Objetivando investigar como a Educação vem se apropriando dos estudos psicológicos e como essa relação entre as disciplinas aparece nas pesquisas em ciências humanas, propôs-se neste artigo, verificar nas publicações da área, como a Educação vem dialogando com a Psicologia. Assim, este artigo realizou um levantamento bibliográfico em duas bases de dados referenciadas na área de ciências humanas (PsycINFO e Web of Science). Os resultados foram organizados em eixos temáticos, nos quais se identificou a predominância de dois eixos: 1) Estudos que utilizam as ferramentas psicológicas para avaliar a efetividade de programas educacionais ambientais específicos; 2) Estudos que procuram resgatar conceitos da psicologia ambiental como indicadores de intervenções na relação pessoaambiente. Nesse sentido, este artigo sugere a apropriação pela educação ambiental dos estudos em Psicologia Ambiental Evolutiva (eixo temático 3), os quais identificam durante todo o ciclo vital as dimensões intervenientes no processo de aprendizagem e constituição do sujeito ecológico.
Aiming to investigate how education comes to appropriating the psychological studies and how this relationship between the disciplines in the research appears in the humanities, it was proposed in this article, check in area publications such as Education is talking to Psychology. Thus, this paper conducted a literature review in two databases referenced in the area of humanities (PsycINFO and Web of Science). The results were organized into themes, in which they identified the predominance of two axes: 1) Studies that use of psychological tools to assess the effectiveness of specific environmental education programs, 2) studies that try to rescue concepts of psychology as indicators of environmental interventions in person-environment relationship. Thus, this article suggests the appropriation for environmental education studies in environmental psychology evolutionary (main theme 3 ), which identified throughout the life cycle dimensions involved in the learning process and constitution of the ecological self.

\footnotetext{
* Psychology and environmental education

${ }^{1}$ Aluna do PPG em Psicologia/UFSC, Endereço para correspondências: Laboratório de Psicologia Ambiental, CFH/UFSC, Campus Universitário, Florianópolis, SC, 88040-970 (lua_sr@yahoo.com.br)

${ }^{2}$ Professora do Programa de Pós-Graduação em Psicologia, Universidade Federal de Santa Catarina, Florianópolis/SC (ariane@cfh.ufsc.br).
} 
Palavras-chave: Educação ambiental - Psicologia ambiental - Interdisciplinaridade
Keywords: Environmental education - Environmental psychology - Interdisciplinary

\section{Introdução}

A literatura especializada em educação e meio ambiente vem crescendo dia nas (GONZÁLEZ-GAUDIANO \& LORENZETTI, 2009). Mas apesar de todos concordarem que é preciso fazer algo a respeito da crise ambiental e que isso exige uma mudança nas atitudes das pessoas, há divergências e disputas entre diversos pontos de vista sobre o que fazer, sobre como fazer, e ainda, sobre quais interesses devem prevalecer na complexa negociação entre os grupos sociais, entre o progresso e a modernidade, que envolvem projetos, visões de mundo e posicionamentos científicos distintos.

$\mathrm{O}$ que parece ser de certa forma consenso nas ciências humanas, entretanto, é a constatação de que o principal responsável pela crise ambiental é o homem. Para Pinheiro (2002) os danos ambientais tem aumentado e se tornado mais evidentes a cada dia, tanto para os cientistas quanto para a população em geral. Em decorrência disso, tem crescido o interesse pelos aspectos psicológicos da relação pessoa-ambiente nas diversas áreas do conhecimento e paralelamente, o interesse em se identificar os motivos de rejeição para com os comportamentos pró-ambientais e as propostas de sustentabilidade pelo homem (CORRAL-VERDUGO \& PINHEIRO, 1999).

A Educação Ambiental, por exemplo, convocada a pensar os problemas concretos da relação pessoa-ambiente, contribuindo para o bem estar geral e para a sobrevivência da espécie humana (UNESCO, 1978), sempre se deparou com a problemática desta relação. Pois constatou que as ações humanas e mais especificadamente as atitudes e comportamentos ecológicos são complexos e multideterminados (CARVALHO, 2006). Uma pessoa pode cultivar uma ideologia ecológica mas, por vários motivos, seguir mantendo condutas nem sempre em conformidade com esses ideais.

Percebeu-se que ao privilegiar a dimensão comportamental da atitude, treinando os sujeitos para ações pró-ambientais programadas, não se estava criando uma atitude ecológica de fato. Ir além de uma aprendizagem comportamental, levando em conta as dimensões afetivas e cognitivas da atitude, é atualmente o desafio enfrentado pela Educação Ambiental que se autodenomina "Crítica" (CARVALHO, 2006).

A EA Crítica entende por atitude ecológica a adoção de um sistema de crenças, valores e sensibilidades éticas e estéticas orientados segundo os ideais 
de vida de um sujeito ecológico, ou seja, como aquele que segue um modo ideal de ser e viver, com modos próprios de pensar o mundo e de pensar a si mesmo e as relações com os outros (CARVALHO, 2006). Ou seja, segue buscando o desenvolvimento de uma compreensão integrada do meio-ambiente em suas múltiplas e complexas relações envolvendo aspectos ecológicos, psicológicos, legais, políticos, sociais, econômicos, científicos, culturais e éticos.

Segundo Reigota (2000), um expoente da educação ambiental brasileira, há várias interpretações sobre o que é Educação Ambiental, o que gera muitas e diferentes práticas educativas em diversos contextos. Para esse autor, as atividades em EA vão desde as intervenções nos clássicos problemas relacionados com o destino do lixo até as práticas de agricultura orgânica e os problemas climáticos globais. Portanto, a diversidade metodológica e conceitual nos estudos de EA é uma característica dessa área.

González-Gaudiano e Lorenzetti (2009) realizaram um estudo para mapear a situação da pesquisa em EA na América Latina. Eles constataram limitados avanços na área com o passar dos anos, marcados por um processo lento e gradual de posicionamento frente ao pensamento norteador crítico da EA. Esses autores discutem que apesar do advento da Educação Ambiental Crítica, ainda existem algumas orientações pedagógicas que concebem a finalidade e a efetividade da educação na mudança de comportamentos de agressão ou indiferença par com o meio ambiente.

Os educadores e pesquisadores da área (SAMPAIO \& GUIMARÃES, 2009) criticam as atividades que ensinam o que fazer e como fazer corretamente, transmitindo uma série de procedimentos ambientalmente corretos. Pois, segundo eles, isso nem sempre garante a formação de uma atitude ecológica, isto é, de um sistema de valores sobre como se relacionar com o ambiente, sistema que será internalizado como uma visão de mundo orientadora dos posicionamentos do sujeito em todos os espaços circundantes de sua vida. Portanto, gerar comportamentos individuais ordeiros, preocupados com a limpeza de uma área ou com a energia elétrica, pode ser socialmente desejável e útil, mas não significa necessariamente que tais comportamentos sejam integrados na formação de uma atitude ecológica cidadã.

A EA Crítica apresenta uma abordagem globalizante de meio ambiente, desenvolvendo-se numa perspectiva crítica, ética e democrática e se propõe a preparar cidadãos que se empenhem na busca de um melhor relacionamento com o mundo, questionadores das causas dos problemas ambientais e que tenham preocupações com os componentes ambientais em suas especificidades e interações, tecendo redes visíveis e invisíveis ao seu redor (GONZÁLEZGAUDIANO \& LORENZETTI, 2009). A compreensão de interdisciplinaridade está embutida nesta EA, assim como seu viés holístico, sua rede de ação múltipla e sua essência de participação individual e coletiva. 
Nesse sentido a interdisciplinaridade entre a Educação Ambiental e a Psicologia é sempre promissora. Pois, também buscando superar as limitações e barreiras às mudanças dos comportamentos pró-ambientais, respondendo a essa demanda, a Psicologia construiu um rico montante de descobertas relativas à experiência, percepções, atitudes e comportamentos sustentáveis. A Psicologia Ambiental (PA) numa compreensão ampla dos estudos da relação pessoa-ambiente busca identificar os modos pelos quais os aspectos sociais e físicos do ambiente influenciam o comportamento das pessoas. De forma transacional, também compreende que as ações das pessoas, por sua vez, afetam os seus entornos (CORRAL-VERDUGO, 2005). A PA considera que de um lado, há um ambiente objetivo, feito de elementos físicos, que afetam as nossas sensações, percepções e ações, e de outro lado, há os seres humanos, como sujeitos sociais, os quais criam e também estão expostos a um ambiente de artefatos culturais, símbolos e convenções (RIVLIN, 2003).

A PA, assim como a EA, valoriza uma abordagem interdisciplinar, ou seja, responde a anseios de outras áreas e partilha, com elas, campos comuns. De maneira geral faz um esforço de cooperação entre disciplinas afins para abordar conjuntamente a investigação ou solução dos problemas humanoambientais concretos (WIESENFELD, 2005).

Segundo Wiesenfeld (2005) os pioneiros da PA colocaram como interesses principais da disciplina: estudar a relação pessoa-ambiente no contexto natural; abordar a dita relação de maneira holística; incorporar diversas perspectivas teóricas em seu estudo; enfatizar a dimensão social da relação humanoambiental; estabelecer vínculos com outras disciplinas interessadas na temática humano-ambiental; e aplicar os conhecimentos obtidos para melhorar a qualidade ambiental e, por conseguinte, a qualidade de vida dos usuários dos ambientes. Também para Pinheiro (2003) uma maneira bastante pertinente de caracterizar a PA é através do exame dos tópicos mais freqüentemente estudados, pois eles identificam o campo de modo mais ou menos consensual.

Duas abordagens dominaram a PA (e ainda prevalecem) desde as suas origens. Uma abordagem privilegiou o estudo dos efeitos ambientais sobre o comportamento. Estudos referentes à percepção ambiental, mapas cognitivos, preferências ambientais, ao efeito da estimulação ambiental sobre o desempenho humano, às relações entre o projeto e o uso de espaços construídos, e às avaliações pós-ocupação são representativos desta abordagem. A segunda tradição hospeda os estudos referentes a como e porquê o comportamento humano afeta o ambiente. Esta inclui a pesquisa sobre conservação e comportamento sustentável, o estudo de crenças ambientais, valores, personalidade e capacidades (entre outros) e a investigação da associação entre variáveis demográficas e comportamento ambientalmente relevante. Igualmente sob esta perspectiva está a relação entre educação ambiental e comunicação, fatores situacionais (tanto normativos quanto físicos) e conservação ambiental (PINHEIRO, 2003). 
Ainda de acordo com Pinheiro (2003), na atualidade, os estudos no campo do compromisso ambiental buscam uma maior contextualização, à medida que incluem bases culturais e históricas dos valores das pessoas, aspectos afetivos, ideologias políticas e visões de mundo. Entende-se que não se pode pensar em resolver problemas ambientais sem vê-los em seu contexto cultural, econômico e social mais amplo (UZZELL, 2004). Perspectiva essa, já anteriormente mencionada, em concordância com a proposta da Educação Ambiental Crítica.

Para a PA, a rejeição das pessoas às atitudes e aos comportamentos próambientais deve ter algo a ver com a percepção ambiental e os valores culturais que dominam a sociedade (UZZELL, 2004). Os ditos indicadores predisposicionais, como as crenças, a satisfação com o lugar, o apego ao lugar, a percepção de risco, as condições ambientais e estilos de vida, são usados pelos indivíduos como referências para executarem suas ações. Nesse sentido, segundo Pinheiro e Pinheiro (2007) deve-se nas pesquisas psicológicas buscar estratégias que diminuam ao máximo as discrepâncias entre o comportamento pró-ambiental e suas medidas predisposicionais, para que os resultados empíricos possam bem servir às intervenções socioecológicas, como no caso da educação ambiental.

Considerando então que boa parte dos psicólogos ambientais está dedicada na tarefa de estudar os indicadores predisposicionais e sua relação com o comportamento humano, a fim de conseguir identificar uma atitude favorável para a preservação ambiental e sugerir intervenções na área educacional, como a EA vem se apropriando desse conhecimento?

Objetivando, portanto, investigar como a Educação vem se apropriando dos estudos psicológicos e como isso aparece nas pesquisas em ciências humanas, propôs-se neste artigo, iniciar um primeiro contato com essa temática, verificando nas pesquisas da área, como a Educação Ambiental vem dialogando com a Psicologia. Assim, este artigo realizou um levantamento bibliográfico em duas bases de dados referenciadas na área de ciências humanas.

O levantamento bibliográfico foi realizado nas bases de dados PsycINFO e Web of Science. A PsycINFO é uma base de dados na área de psicologia, que reúne periódicos publicados pela American Psychological Association, Canadian Psychological Association Journals e Hogrefe Publishing Group. A Web of Science é uma base multidisciplinar que indexa os periódicos mais citados em diversas áreas. As palavras-chave utilizadas na busca foram: environmental psychology and education e environmental education and psychology.

\section{Resultados e discussão}

Entre os artigos encontrados, foram selecionados aqueles que realizaram estudos relacionando conceitos da Psicologia Ambiental com a prática educacional ou ainda que mostravam, terem realizado uma interação entre as duas áreas. 
Na base $P$ sycINFO foram encontrados 38 artigos, sendo excluídos 14 por não atenderem aos critérios de inclusão na pesquisa. Na base Web of Science foram encontrados 51 artigos, desses, apenas 19 artigos atenderam aos critérios de inclusão na pesquisa. No total foram analisados 42 artigos.

\section{Quadro 1}

Pesquisas relacionando Psicologia e Educação Ambiental

\begin{tabular}{|c|c|c|c|}
\hline $\begin{array}{c}\text { Eixo } \\
\text { Temático }\end{array}$ & PsycINFO & Web of Science & Total \\
\hline $\begin{array}{l}\text { 1) Utilizam de } \\
\text { ferramentas } \\
\text { psicológicas } \\
\text { para avaliar } \\
\text { programas } \\
\text { educacionais } \\
\text { ambientais }\end{array}$ & $\begin{array}{l}\text { (CHRISTENSEN; ROWE et al., } \\
\text { 2007) (BOGNER; WISEMAN, } \\
\text { 2002) (BOGNER, 2002) (POL; } \\
\text { VIDAL et al., 2001) (BURRUS- } \\
\text { BAMMEL; BAMMEL et al., } \\
\text { 1988) (FARMER; KNAPP et al., } \\
\text { 2007) (SHARMA, 2009) } \\
\text { (TZAMIR; CHURCHMAN, } \\
\text { 1984; WING; HORTON et al., } \\
\text { 2008) (DELP; BROWN et al., } \\
\text { 2005) (ALEM; MCLEAN, 2005) }\end{array}$ & $\begin{array}{l}\text { (STAGL, 2007) } \\
\text { (MEASHAM, 2006) } \\
\text { (Hassard, 1997) } \\
\text { (SEMKEN; } \\
\text { FREEMAN, 2008) } \\
\text { (PRICE; VINING et } \\
\text { al., 2009) } \\
\text { (ROOMRATANAPU } \\
\text { N, 2001) }\end{array}$ & 17 \\
\hline $\begin{array}{l}\text { 2) Incluem os } \\
\text { estudos que } \\
\text { resgatam } \\
\text { conceitos da } \\
\text { PA como } \\
\text { indicadores de } \\
\text { intervenções } \\
\text { ambientais }\end{array}$ & $\begin{array}{l}\text { (KOGER; SCOTT, 2007) } \\
\text { (VITOUCH, 1993) } \\
\text { (PINHEIRO; PINHEIRO, } \\
\text { 2007) (ATTILA, 2006) } \\
\text { (ZINT, 2002) (MCANDREW, } \\
\text { 1995) }\end{array}$ & $\begin{array}{l}\text { (BADER, 1993) } \\
\text { (KALOF; DIETZ et al., } \\
\text { 1999) (NEVERS; } \\
\text { GEBHARD et al., 1997) } \\
\text { (WORSLEY; } \\
\text { SKRZYPIEC, 1998) } \\
\text { (ZAPF, 2005) } \\
\text { (OSTMAN, 2010) } \\
\text { (NIXON; SAPHORES, } \\
\text { 2009) (IOAN; } \\
\text { MACOVEANU et al., } \\
\text { 2008) (NISBET; GICK, } \\
\text { 2008) (BOWEN; } \\
\text { CHANG et al., 1996) }\end{array}$ & 16 \\
\hline $\begin{array}{l}\text { 3) Relação } \\
\text { entre PA e PD }\end{array}$ & $\begin{array}{l}\text { (ELALI, 2003) (HUSE, 1995) } \\
\text { (HART, 1987) (RIVLIN; } \\
\text { WEINSTEIN, 1984) } \\
\text { (CAMPOS-DE-CARVALHO; } \\
\text { SOUZA, 2008) (FINKEL, } \\
\text { 1975) }\end{array}$ & $\begin{array}{l}\text { (WYLIE; SHEEHY } \\
\text { et al., 1996) } \\
\text { (SCHUMANNHENG } \\
\text { STELER; THOMAS, } \\
\text { 1994) (DURAN- } \\
\text { NARUCKI, 2008) } \\
\end{array}$ & 9 \\
\hline Total & 23 & 19 & 42 \\
\hline
\end{tabular}


Os artigos foram categorizados conforme os eixos temáticos, definidos com base nos temas abordados nas pesquisas encontradas. Identificaram-se 3 eixos temáticos principais: 1) Incluem estudos que utilizam as ferramentas psicológicas para avaliar a efetividade de programas educacionais ambientais específicos; 2) Incluem os estudos que procuram resgatar conceitos da psicologia ambiental como indicadores de intervenções ambientais; 3) Incluem estudos que ao realizarem um diálogo entre a PA e a Psicologia do Desenvolvimento (PD) também propõem alternativas para a construção de um sujeito ecológico. A quantidade de artigos encontrados em cada eixo temático pode ser observada no Quadro 1, bem como as diferenças em relação à distribuição nos eixos dos artigos encontrados na PsycINFO e na Web of Science.

No Quadro 1 pode-se observar os temas encontrados, o número de artigos em cada um e as referências dos estudos em cada tema. Ao analisar esses resultados percebeu-se que houve equilíbrio de artigos que utilizam a Psicologia como ferramenta avaliativa da efetividade dos programas educativos e daqueles que utilizam os conceitos como indicadores para os programas. Com relação às diferenças entre as bases de dados, observou-se uma predominância do eixo 2, que trata da relação entre os conceitos psicológicos e as problemáticas ambientais, na base de dados Web of Science, enquanto na base $P$ sycINFO houve um predomínio dos artigos que utilizam da Psicologia como ferramenta de avaliação dos processos de aprendizagem na EA.

No primeiro eixo temático identificou-se pesquisas preocupadas em avaliar a efetividade de programas ambientais por meio de medidas psicológicas. Esses estudos buscavam mensurar se a participação do sujeito em uma determinada atividade educativa tinha influência causal sobre mudanças em crenças e atitudes ecológicas. Farmer e colaboradores (2007), por exemplo, examinaram os efeitos a longo prazo de um passeio a uma reserva ambiental (vivência) por crianças da quarta série do ensino fundamental. Seus resultados sugerem que, um ano depois da vivência, muitos alunos ainda se lembravam do que tinham visto e ouvido e demonstravam um comprometimento pró-ambiental, os pesquisadores então discutem a efetividade de programas educativos que investem nas experiências práticas das crianças com os ambientes.

Semkem e Freeman (2008), por exemplo, defendem que os estudos de apego ao lugar reforçam a constatação da importância dos programas educacionais contextualizados. Essas pesquisas são exemplos da discussão em torno da dicotomia local/global (HASSARD, 1997). Para Uzzell (2004), estudos que diferenciam a influência dos aspectos locais e globais são cruciais no sentido de se entender a percepção pública e as atitudes relacionadas a problemáticas ambientais, assim como entender seus comportamentos subseqüentes. 
Stagl (2007) constata que a relação dos programas de EA com as clássicas teorias de aprendizagem é bastante fraca. Este autor defende que uma melhor compreensão dos processos de aprendizagem poderia fornecer pistas importantes para recomendações de programas e políticas públicas voltadas para o desenvolvimento sustentável, pois a maneira como aprendemos sobre o nosso planeta reflete-se na forma como interagimos com ele.

Assim, observando a interação de crianças e adolescentes com o lugar de moradia, Measham (2006) chama atenção para a valorização das políticas ambientais cujas intervenções buscam resgatar a vivência no lugar de origem e moradia dos sujeitos, através do aprendizado nas tradições locais, bem como, no aprender com os eventos extremos, tais como incêndios e inundações. Apostam que se torna muito mais fácil para as pessoas entenderem os problemas ambientais locais e envolverem-se nestes na medida em que podem perceber os efeitos benéficos ou não de suas ações no espaço.

Identificaram-se também, alguns estudos de casos que relatam intervenções realizadas em comunidades em situação de risco ambiental (ROOMRATANAPUN, 2001; ALEM \& MCLEAN, 2005; DELP et al., 2005; WING et al., 2008). Discutem que através de técnicas que resgatam a percepção de risco dos envolvidos na comunidade é possível avaliar a efetividade de programas de intervenção que visam identificar quais os meios pelos quais os indivíduos, em um contexto social, constroem o ambiente de modo a compreender e lidar com um estado de coisas ameaçador.

No segundo eixo temático as pesquisas relacionam conceitos psicológicos como percepção de risco, cognição, identidade e apego ao lugar, crenças, valores, condutas e atitudes às problemáticas ambientais, principalmente ao desejado comportamento sustentável, trazendo resultados que sugerem alternativas de suporte teórico metodológico para projetos em educação. Estes estudos acreditam que a PA pode contribuir com a criação de uma sociedade sustentável oferecendo ajuda para a gestão da crise ambiental (MCANDREW, 1995; ATTILA, 2006; KOGER \& SCOTT, 2007). Pinheiro e Pinheiro (2007) ao estudarem a pré-disposição para o comportamento de "cuidado ambiental" identificaram uma ponte de ligação entre a Psicologia e a Educação e sugerem que os resultados das investigações psicológicas podem ser ferramentas importantes para as ações educativas.

Worsley e Skzypiec (1998), por exemplo, investigaram a atitude ecológica de 958 alunos do ensino médio em 32 países através da aplicação de questionários que resgatavam a dimensão cognitiva e afetiva das crenças ambientais. Seus resultados indicaram que apesar dos estudantes alegarem preocupação com o meioambiente eles também demonstraram bastante pessimismo em relação as soluções para os problemas ambientais. Os autores discutem se esse pessimismo não estaria relacionado com o tipo de educação a que eles estavam sendo submetidos. 
Com as mesmas indagações sobre a qualidade da educação, Zint (2002) investiga a atitude de professores de ciências buscando prever a intenção destes em agir de forma respeitosa com o meio-ambiente, alertando para a necessidade de uma maior formação profissional dos educadores.

Ainda buscando ferramentas para a educação ambiental, os estudos de Ostman (2010) e Nevers e colaboradores (1997) sugerem uma associação entre o desenvolvimento moral do indivíduo e a relação do homem com o meioambiente, incluindo no ensino da filosofia moral a relação humana com outros objetos, como as plantas, os animais e o ecossistema. Ilustrando essa idéia, Nevers e colaboradores (1997) traz como pesquisa um exemplo de investigação com crianças e adolescentes avaliando a aquisição de valores morais e as atitudes relevantes de respeito com a natureza em diferentes faixas etárias.

Por fim, agrupamos no último eixo temático, as pesquisas em Psicologia Ambiental Evolutiva, ou seja, que trazem uma relação entre a PA e a PD contribuindo para as questões de aprendizagem. De maneira geral esses estudos se caracterizam por contemplarem os acontecimentos que se manifestam durante o ciclo vital no processo relacional entre o indivíduo e seu entorno imediato ou mais remoto. Aqui neste artigo eles foram incluídos nos resultados por resgatarem a dimensão física dos ambientes de aprendizagem, lugares construídos onde ocorre a educação ambiental formal.

Segundo Correa e Ruiz (2008) a terminologia Psicologia Ambiental Evolutiva surgiu da necessidade da PA agrupar as pesquisas em sua área, cujo tema de interesse principal fosse o desenvolvimento humano. Questões como os mecanismos através dos quais o meio contribui na dimensão cognitiva, social, emocional e comportamental do ser humano; as propriedades dos diferentes entornos contextualizados como ambientes de desenvolvimento humano e que respondam as necessidades evolutivas de cada etapa do ciclo vital, são alguns dos tópicos que caracterizam a Psicologia Ambiental Evolutiva.

Para Schumannhengsteler e Thomas (1994), durante o ciclo vital humano, informação combinada com atividades são fundamentais para o desenvolvimento da atitude ecológica. Estes autores realizaram três estudos com crianças do ensino fundamental, a fim de resgatar o conhecimento dessas sobre o cuidado com o ambiente. No primeiro estudo através de entrevistas e evocações livres os resultados mostraram uma relação entre a idade da criança e o conhecimento, bem como, um efeito da familiaridade com a situação ecológica proposta, por exemplo, as crianças sabiam mais sobre a separação de lixo do que sobre a reciclagem. No segundo estudo foram realizadas entrevistas clínicas baseadas nas teses piagetianas sobre o desenvolvimento cognitivo, objetivando separar o conhecimento declarado ("O que é ecologicamente prejudicial?") e as teorias ingênuas sobre as relações de causalidade ("Por que é ecologicamente prejudicial?"). Para todas as faixas etárias, 
eles encontraram uma compreensão das relações de causalidade muito menor do que o conhecimento declarado. Num terceiro estudo eles examinaram se a experiência cotidiana com a proteção do ambiente tinha efeitos sobre a qualidade das informações. Eles concluíram que a experiência cotidiana é uma condição necessária para o conhecimento sobre as relações de causalidade em crianças menores, mas não nos mais velhos, discutindo as implicações destes resultados para a EA em crianças pré-escolares.

A maioria dos estudos encontrados na busca bibliográfica do último eixo temático são diálogos teóricos e metodológicos entre as áreas de psicologia, educação, planejamento ambiental e design, resgatando a relação entre o desenvolvimento psicológico da criança e a paisagem física dos seus contextos imediatos de desenvolvimento, como por exemplo, a escola (RIVLIN \& WEINSTEIN, 1984; ELALI, 2003; CAMPOS-DE-CARVALHO \& SOUZA, 2008). Dentro desta linha teórica de pesquisa encontramos como principal referencial teórico o modelo de desenvolvimento psicológico de Bronfenbrenner (2001), pois este autor prioriza a compreensão do desenvolvimento infantil atrelado ao contexto no qual ocorre, identificando nos entornos, imediatos e remotos, variáveis (culturais, sociais, biológicas) que afetam e explicam o desenvolvimento sob uma perspectiva sistêmica e transacional, ou seja, sob uma perspectiva bioecológica do desenvolvimento humano.

\section{Considerações finais}

Ao se buscar resgatar a possível interdisciplinaridade entre a PA e a EA, ou seja, buscando os indicadores de uma práxis unificadora nos relatos de pesquisas, pode-se constatar que apesar de haver ainda uma tendência interdisciplinar em utilizar os instrumentos da Psicologia apenas como ferramenta de avaliação da efetividade dos programas educativos, já há uma busca também em aprofundar este diálogo entre as disciplinas, através da apropriação, por ambas as partes, de conceitos e referenciais teóricos construídos em função da busca comum de resposta para o desenvolvimento de um sujeito ecológico.

Dessa forma é promissora a idéia de se aliar a esse diálogo, ainda mais estudos que procurem resgatar também as dimensões bioecológicas do desenvolvimento humano, durante todo o ciclo vital do sujeito. Pois, apesar de ser uma idéia coerente, ela ainda é escassa nos periódicos científicos, como se pode observar. No sentido de contribuir com o avanço de uma construção teórica, portanto, sugere-se mais estudos da Psicologia Ambiental Evolutiva que busquem identificar princípios norteadores ou indicadores predisposicionais para o comportamento pró-ambiental. 
Nesse sentido são relevantes os estudos de Kahn e Lourenço (2002) que sugerem a aplicabilidade das teses piagetianas na compreensão, do que estes autores chamam, da construção do raciocínio ecológico-moral na infância. Objetivando analisar como e quando surge nas crianças a idéia de respeito ao meio ambiente como um imperativo moral, assim como evidenciar os raciocínios sobre os quais ele se estabelece, realizaram estudos através da técnica da entrevista com dilemas morais. Os autores concluíram, que já no início do ensino fundamental, muitas crianças já parecem convencidas de que temos uma obrigação moral para com o meio ambiente, ainda que esteja baseada inicialmente em raciocínios antropocêntricos ou em uma visão pouco elaborada do prejuízo à natureza. E que apesar de haverem aspectos da própria natureza, do contexto, que ajudam a dar origem ao raciocínio moral ambiental nas crianças, os estudos constatam que em diferentes culturas, o raciocínio moral biocêntrico (aquele que considera o valor intrínseco da natureza ou seus direitos) surge de fato (ou mais plenamente) apenas em adolescentes e adultos.

Enfim, as teorias psicológicas evolutivas do desenvolvimento humano, buscam considerar no processo de crescimento do sujeito, fatores como, os paradigmas dominantes, os valores ideológicos, políticos, morais, culturais, artísticos, sociais, econômicos e religiosos. Fatores estes que para os estudiosos do desenvolvimento subsidiam uma melhor compreensão da construção do raciocínio sobre o meio-ambiente. Portanto, estes estudos psicológicos chamam a atenção para a importância de uma EA que inclua em seus programas uma reflexão teórica sob uma perspectiva do tipo desenvolvimentista e construtivista, ou seja, que leve em consideração que as concepções e valores ambientais dos sujeitos refletem formas organizadas e generalizadas de pensamento ecológicomoral construídas pela sua contínua interação com o seu meio social.

Dessa forma, sugere-se que através da compreensão do desenvolvimento humano integral seria possível pensar em atividades e programas educacionais voltados para a formação continuada de sujeitos ecológicos, promovendo competências nas crianças e jovens, mas também em adultos e idosos participantes ativos nas tomadas de decisão relativas aos problemas ambientais durante todo o ciclo vital humano.

\section{Referências bibliográficas}

ALEM, L. \& MCLEAN, A. Supporting learning and information sharing in natural resource management with technologies for electronic documents. Supporting e-learning with technologies for electronic documents, p. 7, 2005.

ATTILA, V. Psychology for sustainability. Magyar Pszichológiai Szemle, 61(1): 187-206, 2006. 
BADER, R. Overcoming false public beliefs - Possible strategies for improving environmental risk literacy. Toxicological and environmental chemistry, 40(14): 275-88, 1993.

BOGNER, F. The influence of a residential outdoor education programme to pupil's environmental perception. European journal of Psychology of education, 17(1): 19-34, 2002.

BOGNER, F. \& WISEMAN, M. Environmental perception of French and some Western European secondary school students. European Journal of Psychology of Education, 17(1): 3-18, 2002.

BOWEN, W.M.; CHANG, C.C. \& HUANG, Y.K.. Psychology and global environmental priorities in Taiwan: A psychometric comparison of two learning models. Journal of environmental psychology, 16(3): 259-68, 1996.

BRONFENBRENNER, U. Human Development, Bioecological Theory of. In: International Encyclopedia of the Social \& Behavioral Sciences. Elsevier Science Ltd. Human Development. Pp. 6963-70, 2001.

BURRUS-BAMMEL, L.; BAMMEL, G. \& KOPITSKY, K. Content analysis: technique for measuring attitudes expressed in environmental education literature. Journal of environmental education, 19(4): 32-7, 1988.

CAMPOS-DE-CARVALHO, M. \& SOUZA, T. Environmental and Developmental Psychology and Early Childhood Education: is there a possible integration? Paidéia (Ribeirão Preto), 18: 25-40, 2008.

CARVALHO, I.C.D.M. Educação ambiental: a formação do sujeito ecológico. São Paulo: Cortez, 2006.

CHRISTENSEN, A.; ROWE, S. \& NEEDHAM. M. Value orientations, awareness of consequences, and participation in a whale watching education program in Oregon. Human dimensions of wildlife, 12(4): 289-93, 2007.

CORRAL-VERDUGO, V. Psicologia Ambiental: objeto, "realidades" sóciofísicas e visões culturais de interações ambiente-comportamento. Psicologia USP, 12(16): 71-87, 2005.

CORRAL-VERDUGO, V. \& PINHEIRO, J.Q. Condições para o estudo do comportamento pró-ambiental: SciELO Brasil, 4, 1999.

CORREA, N. \& RUIZ, C. Psicología Ambiental Evolutiva: construyendo un punto de encuentro. Medio ambient. comport. hum., 9: 1-11, 2008. 
DELP, L.; BROWN, M. \& DOMENZAIN, A. Fostering youth leadership to address workplace and community environmental health issues: A universityschool-community partnership. Health promotion practice, 6(3): 270, 2005.

DURAN-NARUCKI, V. School building condition, school attendance, and academic achievement in New York City public schools: A mediation model. Journal of environmental Psychology, 28(3): 278-86, 2008.

ELALI, G. O ambiente da escola-o ambiente na escola: uma discussão sobre a relação escola-natureza em educação infantil. Estud. Psicol. (Natal), 8(2), 2003.

FARMER, J.; KNAPP, D. \& BENTON, G. An elementary school environmental education field trip: Long-term effects on ecological and environmental knowledge and attitude development. The journal of environmental education, 38(3): 33-42, 2007.

FINKEL, C.L. The supportive environment: A new dimension in meetings. Training \& development journal, 25-36, 1975.

GONZÁLEZ-GAUDIANO, E. \& LORENZETTI, L. Investigação em educação ambiental na América Latina: mapeando tendências. Educação em Revista, 25(3): 191-211, 2009.

HART, R. Environmental psychology or behavioral geography? Either way it was a good start. Journal of environmental psychology 7(4): 321-29, 1987.

HASSARD, J. Teaching students to think globally. Journal of humanistic Psychology, 37(1): 24-63, 1997.

HUSE, D. Restructuring and the physical context: Designing Learning Environments, 12(3): 290-310, 1995.

IOAN, C.C.; MACOVEANU, M. \& ROBU, B. The Gorgon method as a tool in sustainable behavior and development. Environmental engineering and management journal, 7(6): 781-84, 2008.

KAHN, P.H. \& LOURENCO, O. Water, air, fire, and earth - A developmental study in Portugal of environmental moral reasoning. Environment and Behavior, 34(4): 405-30, 2002.

KALOF, L.; DIETZ, T.; STERN, P.C. \& GUAGNANO, G.A. Social psychological and structural influences on vegetarian beliefs. Rural Sociology, 64(3): 500-11, 1999.

KOGER, S. \& SCOTT, B. Psychology and environmental sustainability: A call for integration. Teaching of Psychology, 34(1): 10-8, 2007. 
MCANDREW, F.T. What is "environmental psychology" and what can it offer to students concerned about global environmental issues? Council on undergraduate research quarterly, 16: 89-92, 1995.

MEASHAM, T.G. Learning about environments: The significance of primal landscapes. Environmental management, 38(3): 426-34, 2006.

NEVERS, P.; GEBHARD, U. \& BILLMANN-MAHECHA, E. Patterns of reasoning exhibited by children and adolescents in response to moral dilemmas involving plants, animals and ecosystems: Routledge. 26: 169-86, 1997.

NISBET, E.K.L. \& GICK, M.L. Can health psychology help the planet? applying theory and models of health behaviour to environmental actions. Canadian Psychology-Psychologie Canadienne, 49(4): 296-303, 2008.

NIXON, H. \& SAPHORES, J.D.M. Information and the decision to recycle: results from a survey of US households. Journal of environmental planning and management, 52(2): 257-77, 2009.

OSTMAN, L. Education for sustainable development and normativity: a transactional analysis of moral meaning-making and companion meanings in classroom communication. Environmental education research, 16(1): 75-93, 2010.

PINHEIRO, J. Comprometimento ambiental: perspectiva temporal e sustentabilidade. In: J.G. Martínez \& S.M. Doménech (Eds.). Temas selectos de psicologia ambiental. Pp. 463-83. México: UNAM - GRECO - FUNDACIÓN UNILIBRE, 2002.

PINHEIRO, J. Psicologia ambiental brasileira no início do século XXI: sustentável? In: D.H. Yamammoto \& V.V. Gouveia (Eds.). Construindo a Psicologia brasileira: desafios da ciência e prática psicológica. Pp. 279-313. São Paulo: Casa do Psicólogo, 2003.

PINHEIRO, J. \& PINHEIRO, T. Cuidado ambiental: ponte entre psicologia e educação ambiental? Psico, 38(1), 2007.

POL, E.; VIDAL, T. \& ROMEO, M. Supuestos de cambio de actitud y conducta usados en las campanas de publicidad y los programas de promocion ambiental. El modelo de las 4 esferas Assumptions of attitudinal and behavioural change in advertising campaigns and environmental marketing. The fourspheres model. Estudios de Psicologia, 22(1): 111-26, 2001.

PRICE, E.A.; VINING, J. \& SAUNDERS, C.D. Intrinsic and Extrinsic Rewards in a Nonformal Environmental Education Program. Zoo Biology, 28(5): 36176, 2009. 
REIGOTA, M. Educação ambiental: fragmentos de sua história no Brasil. In: F.O. Noal et al. (Eds.). Tendências da educação ambiental brasileira. P. 263. Santa Cruz do Sul: EDUNISC, 2000.

RIVLIN, L. \& WEINSTEIN, C. Educational issues, school settings, and environmental psychology. Journal of environmental psychology, 4(4): 347-64, 1984.

RIVLIN, L.G. Olhando o passado e o futuro: revendo pressupostos sobre as inter-relações pessoa-ambiente. Estud. Psicol. (Natal)8: 215-20, 2003.

ROOMRATANAPUN, W. Introducing centralised wastewater treatment in Bangkok: a study of factors determining its acceptability. Habitat international, 25(3): 359-71, 2001.

SAMPAIO, S.M.V.D. \& GUIMARÃES, L.B. Educação ambiental: tecendo trilhas, escriturando territórios. Educação em revista, 25(3): 353-68, 2009.

SCHUMANNHENGSTELER, R. \& THOMAS, J. What do children know about environmental-protection. Psychologie in erziehung und unterricht, 41(4): 249-61, 1994.

SEMKEN, S. \& FREEMAN, C.B. Sense of place in the practice and assessment of place-based science teaching. Science education, 92(6): 1042-57, 2008.

SHARMA, A. Interdisciplinary industrial ecology education: recommendations for an inclusive pedagogical model. Asia pacific journal of education, 29(1): 75-85, 2009.

STAGL, S. Theoretical foundations of learning processes for sustainable development. International journal of sustainable development and world eco$\log y$, 14(1): 52-62, 2007.

TZAMIR, Y. \& CHURCHMAN, A. Knowledge, ethics, and environment: Behavior studies in architectural education. Environment and Behavior, 16(1): 111, 1984.

UNESCO. Conferência intergovernamental sobre Educação Ambiental. C. E. C. Tibilisi (URSS): Organização das Nações Unidas para a Educação, 1978.

UZZELL, D. A psicologia ambiental como uma chave para mudar atitudes e ações para com a sustentabilidade. In: E.T.D.O. Tassara et al. (Eds.). Psicologia e ambiente. Pp. 363-88. São Paulo: EDUC, 2004.

VITOUCH, O. Environmental Psychology: Moving toward the principles of responsibility. Studia Psychologica, 35(4-5): 343-46, 1993. 
WIESENFELD, E. A Psicologia Ambiental e as diversas realidade humanas. Psicologia USP, 16(1/2): 53-69, 2005.

WING, S.; HORTON, R; MUHAMMAD, N.; GRANT, G.; TAJIK, M. \& THU, K. Integrating epidemiology, education, and organizing for environmental justice: community health effects of industrial hog operations. American journal of public health, 98(8): 1390, 2008.

WORSLEY, A. \& SKRZYPIEC, G. Environmental attitudes of senior secondary school students in South Australia. Global environmental change-human and policy dimensions, 8(3): 209-25, 1998.

WYLIE, J.; SHEEHY, N.; MCGUINNESS, C. \& ORCHARD, G. Children's cognitions: Issues for global environmental education. Irish journal of Psychology, 17(4): 310-26, 1996.

ZAPF, M.K. The spiritual dimension of person and environment - Perspectives from social work and traditional knowledge. International social work, 48(5): 633, 2005.

ZINT, M. Comparing three attitude-behavior theories for predicting science teachers' intentions. Journal of research in science teaching, 39(9): 819-44, 2002. 